\title{
Charged Colloidal Rods Out of Equilibrium
}

\author{
Kyongok Kang \\ Forschungszentrum Juelich, ICS-3, Soft Condesed Matter, Juelich, Germany \\ Email: k.kang@fz-juelich.de
}

Received 28 October 2014; revised 26 November 2014; accepted 26 December 2014

Copyright (C) 2015 by author and Scientific Research Publishing Inc.

This work is licensed under the Creative Commons Attribution International License (CC BY). http://creativecommons.org/licenses/by/4.0/

(c) †) Open Access

\section{Abstract}

This article is a comprehensive overview of the ongoing research of the author on charged colloidal rods out of equilibrium, under external electric fields and at high concentrations around the glass transition. The suspensions of fd-virus particles are used as a model system for charged colloidal rods, which exhibit several disorder-order (and liquid-crystalline) phase transitions. When a low AC electric field is applied to suspensions in isotropic-nematic coexistence concentration, with frequencies that are sufficiently low to polarize the electric double layer and the layer of condensed ions, various phases/states are induced: a chiral nematic, a dynamical state where nematic domains persistently melt and form, and a uniform homeotropic phase. A point in the field-amplitude versus frequency diagram, where various transitions lines meet, can be identified as a non-equilibrium critical point. Without an electric field, at high concentrations of charged fd-rods, various self-assembled orientation textures are found beyond the isotropic-nematic coexistence regions, and a glass transition is observed on approach and within the glass state that are probed. The presented system exhibits transient behaviors of repulsive glasses and slow dynamics out of equilibrium.

\section{Keywords}

Electric Field Phase/State Diagram, Critical Exponents, Orientation Textures, Glass Transition, Charged Fibrous Viruses

\section{Introduction}

This paper presents results on the non-equilibrium behavior of suspensions of charged colloidal rods under low-frequency external AC electric fields, as well as at relatively high concentrations where a glass state is formed. Various phases/states are induced by an external electric field, for low frequencies less than a few $\mathrm{kHz}$ [1] [2]. As a model system of charged colloidal rods, suspensions of charged fibrous viruses (fd) are used at a low ionic strength corresponding to a Tris/HCl buffer solution of $0.16 \mathrm{mM}$ where the Debye length is $27 \mathrm{~nm}$. 
The particles of fd-virus have a length of $880 \mathrm{~nm}$, a core thickness of $6.8 \mathrm{~nm}$, while the persistence length is $2500 \mathrm{~nm}$. These rods are highly charged, carrying a total charge of about 8800 (negative) elementary charges [3], of which about $80 \%-90 \%$ is compensated by condensed ions. For frequencies below a few $\mathrm{kHz}$, field-induced polarization of the diffusive electric double layer as well as the layer of condensed ions occurs [4] [5], and electro-osmotic flow is induced. The electric field thus induces direct interactions through charge polarization and hydrodynamic interactions through electro-osmotic flow. As will be discussed, the polarization of the layer of condensed ions is accompanied by a cyclic association/dissociation of these ions. These phenomena lead to several field-induced phases/states in suspensions that are in isotropic-nematic coexistence concentration without the external field. In particular, the microscopic origin of a dynamical state, where small nematic domains (of 30 um in size) melt and form, is explained on the basis of a limit cycle of the field-induced association/dissociation of condensed ions [6]. At very low frequencies, electrode polarization due to the buildup of charges at the ITO electrode surfaces becomes important. A theory is discussed to calculate the true bulk electric field strength within the suspension, away from the electrodes, and thus correct for electrode polarization.

Without an external electric field, at higher concentrations, a glass transition occurs far above the two-phase isotropic-nematic coexistence regions [7] [8]. Dynamic light scattering experiments will be discussed which reveal particle arrest, while image-time correlation shows freezing of the orientation texture dynamics, at the same concentration. This glass transition is attributed to particle caging due to strongly overlapping electric double layers. In this short paper, only reference is made to the papers where the original work is described, in which the many references that are relevant to these topics can be found.

\section{Electric-Field Induced Phase Transitions}

\subsection{Electric Phase/State Diagram}

First, depolarized morphologies are observed, depending on the applied field amplitude and frequency, with a homemade electric-cell, under an inverted microscope (Axiovert CFL 40, Carl Zeiss), shown in Figure 1. Local orientation ordering can be observed as bright or dark regions of textures of samples in between two crossed polarizers are regions. The electrical cell consists of two optical, flat ITO electrodes, with a spacing of typically 1.5 $\mathrm{mm}$. The several phases and states are characterized by means of various experimental methods, such as the image-time correlation, dynamic light scattering, and electric birefringence [9] [10]. The various phases and states are shown in Figure 2, in the applied field amplitude versus frequency plane. Solid lines indicate sharp transi-

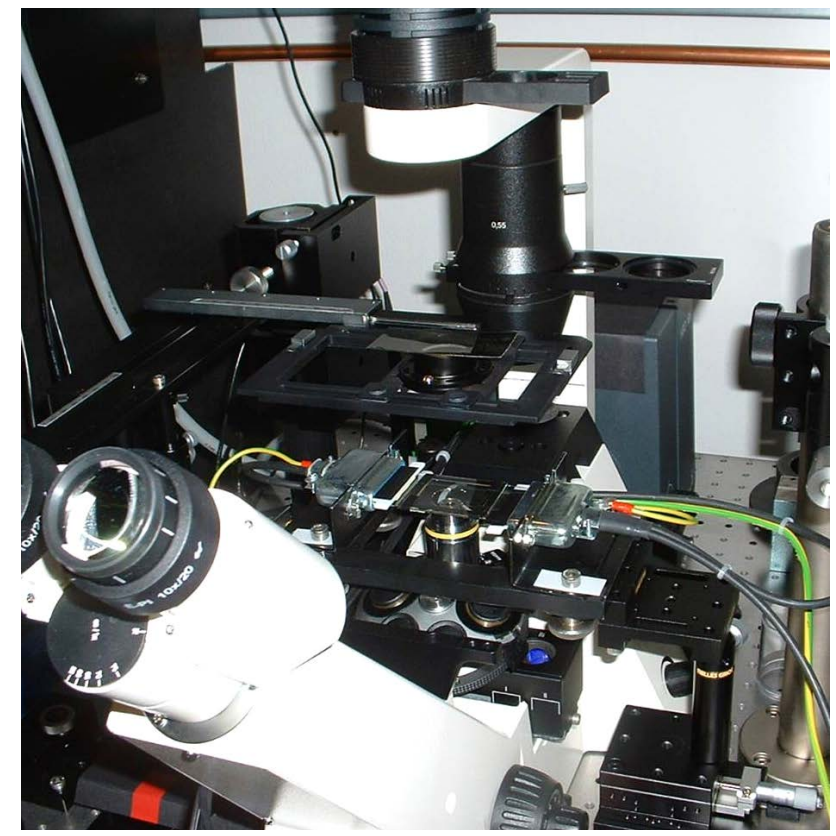

Figure 1. The electrical cell in an inverted microscope with which depolarized images are recorded. 


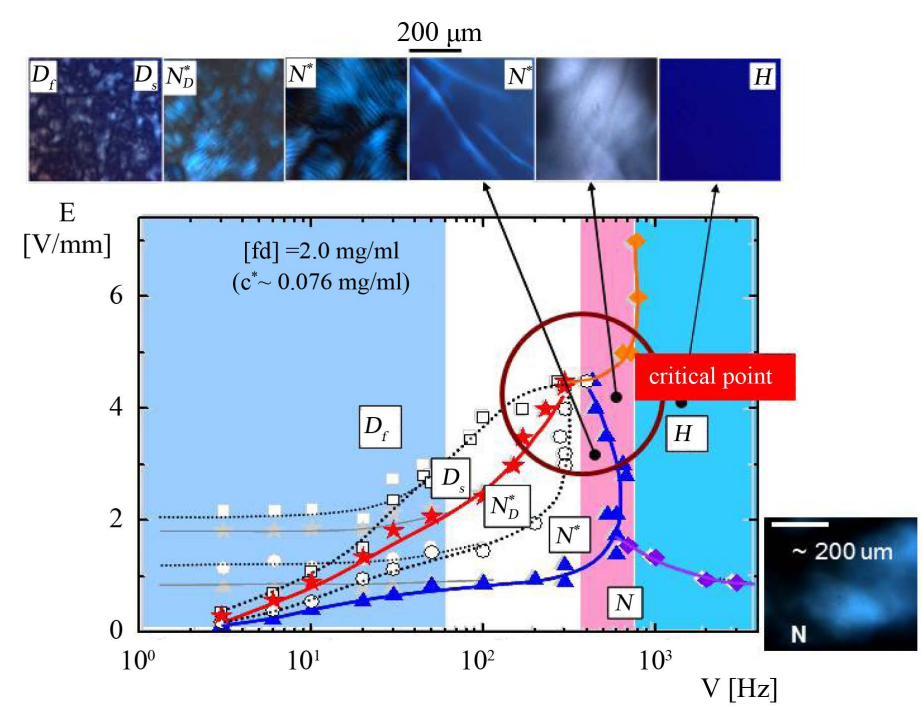

Figure 2. Electric-field phase/state diagram of charged fibrous viruses (fd) in the field amplitude versus frequency diagram, together with depolarized images of the various phases and states: $\mathrm{N}$-isotropic/nematic coexistence, $N^{*}$-chiral-nematic, $N_{D}^{*}$-disconnected chiral-nematic phase, $D_{s}, D_{f}$-slow, fast dynamical state, and $H$-homeotropic phase.

tions while dotted lines are gradual transitions. The fd-concentration is chosen within the two-phase isotropicnematic coexistence region (without the field). Top of Figure 2 shows the corresponding depolarized morphologies of various phases and states, and the phase/state diagram that is in the field-amplitude versus frequency plane. The left blue region in this phase/state diagram indicates the low frequencies (smaller than $60 \mathrm{~Hz}$ ) where electrode polarization is important. The upper phase transition lines in this region are the measured transition lines, while the corresponding lower lines are the transition lines corrected for electrode polarization. A depolarized image of the isotropic-nematic coexistence in the absence of the field is shown in the right lower image in Figure 2. Note that the boundaries of the nematic domains, in the absence of the external electric field, are quite fuzzy. This phase persists up to small field amplitudes, and is denoted as the $N$-phase. On increasing the field strength for frequencies below about $600 \mathrm{~Hz}$, the nematic domains become chiral (the $N^{*}$-phase), which become smaller and disconnect upon entering the $N_{D}^{*}$-phase. Upon further increase of the field amplitude, a sharp transition to a dynamical state is observed (the $D_{s}$-state), where nematic domains persistently melt and form on a time scale of typically tens of seconds. The melting and forming becomes faster when the field amplitude is further increased, and levels of to about 2 seconds in the $D_{f}$-state. At high frequencies, about $600 \mathrm{~Hz}$, the $\mathrm{N}$-phase becomes homogeneous on increasing the field amplitude (the $H$-phase). The pink region indicates the region within the $H$-phase, close to the $N^{*}-H$-transition line, where pre-transitional, transient and blurry domains are exist. The depolarized images of these various phases/states are shown in Figure 2. In the following, the characteristics of these various phases and states will be discussed.

\subsection{Field-Induced Criticality}

There is a point in the phase/state diagram in Figure 2 (encircled in red) where several transition lines meet, seemingly similar to a critical point in equilibrium phase diagrams, which can be distinguished as low-and highfrequency electric fields [2]: at low-frequency, two sharp phase transitions of entering the chiral-nematic $N^{*}$ phase (as the blue solid line) and dynamical states (as the red solid line), however relatively at high frequencies, above a frequency of about $400 \mathrm{~Hz}-1 \mathrm{kHz}$, only homeotropically aligned $H$-phase is stabilized, along the electric field direction (see the most right blue region). Also in between, there is a field-induced critical point as a red circle, where the multi-phases/states meet. The pink region is indicated for the dynamic instability that has shown transient solitary waves, on approaching the critical field amplitude by lowering the frequency. In particular, to present the frequency responsive microscopic dynamics of charged rods in the non-equilibrium, dynamic light scattering is used to characterize the microscopic, particle dynamics on approach of this "non-equilibrium 
critical point” from the side of the $H$-phase [10], and an image-time correlation technique is used to characterize the time scale on which the dynamics of melting and forming of small nematic domains in the dynamical states proceeds [11]. Both methods revealed critical slowing down on approach of the critical point, for different pathways of field amplitude and frequency, while a discontinuity in the microscopic dynamics is observed at the $N_{D}^{*}-D_{s}$-transition line, in the phase/state diagram. For obtaining true field-induced phases, a steady non-critical back ground value $\tau_{b}$, is subtracted from the measured value, in Equation (1), where the divergence of the observable is shown with the external parameter.

Then $\mu_{\tau}^{v}$ is denoted as specifically for the exponent power of a time with an applied frequency parameter. The brief summary of founded field-induced critical exponents is shown in Figure 3. The time scale on which melting and forming occurs in the dynamical state diverges on approach of the $N_{D}^{*}-D_{s}$-transition line and the critical point. For example,

$$
\tau(v)-\tau_{b} \approx\left(1-\frac{v}{v_{c}}\right)^{-\mu_{\tau}^{v}} .
$$

where $\mu_{\tau}^{v}=0.65+/-0.15$ is the critical exponent where the frequency is varied. The maximum domain size also diverges at the critical point. Similarly, for the domain size, similarly, the exponent $\mu_{d}^{E}$ is the exponent power of the domain size with varying the applied field amplitude, shown in Equation (2).

$$
d(E)-d_{b} \approx\left(\frac{E}{E_{c}}-1\right)^{-\mu_{d}^{E}} .
$$

where $\mu_{d}^{E}=1.29+/-0.15$ is the critical exponent for the domain size on approach of the critical point by lowering the field amplitude, and where $\mathrm{db}$ is the non-critical background domain size. An exponential divergence is found on approach of the critical point by lowering the field amplitude at the critical frequency (with an exponent $1.39 \pm 0.18$ ), and by increasing the frequency at the critical field amplitude (with an exponent of $0.65 \pm$ 0.15). A logarithmic divergence is also found on off-critical approach of the $N_{D}^{*}-D_{s}$-transition line [11].

\subsection{In-Situ Electric Field Small Angle Dynamic Light Scattering}

The collective particle dynamics is probed by means of a homemade small angle dynamic light scattering (DLS) set up, a photograph of which is given in the left panel of Figure 4. The incident beam is vertically aligned (from bottom to top), and the sample stage is situated in the middle, with a micrometric adjustment. For the electric field, the same electric cell is used as for the image-time correlation measurements (within the red circle

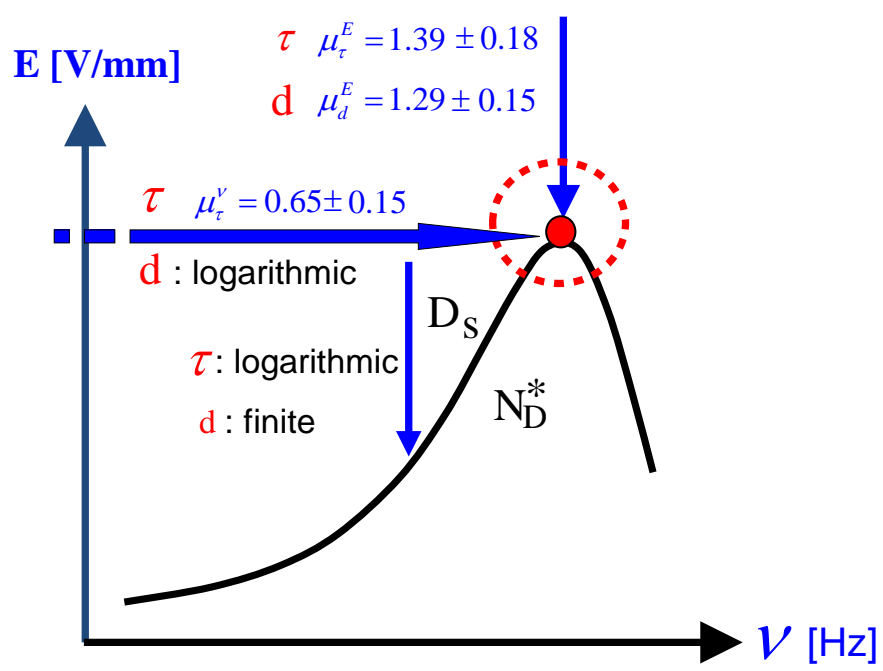

Figure 3. Summary of field-induced critical behavior of the characteristic time for melting and forming of nematic domains and the maximum nematic domain size. 


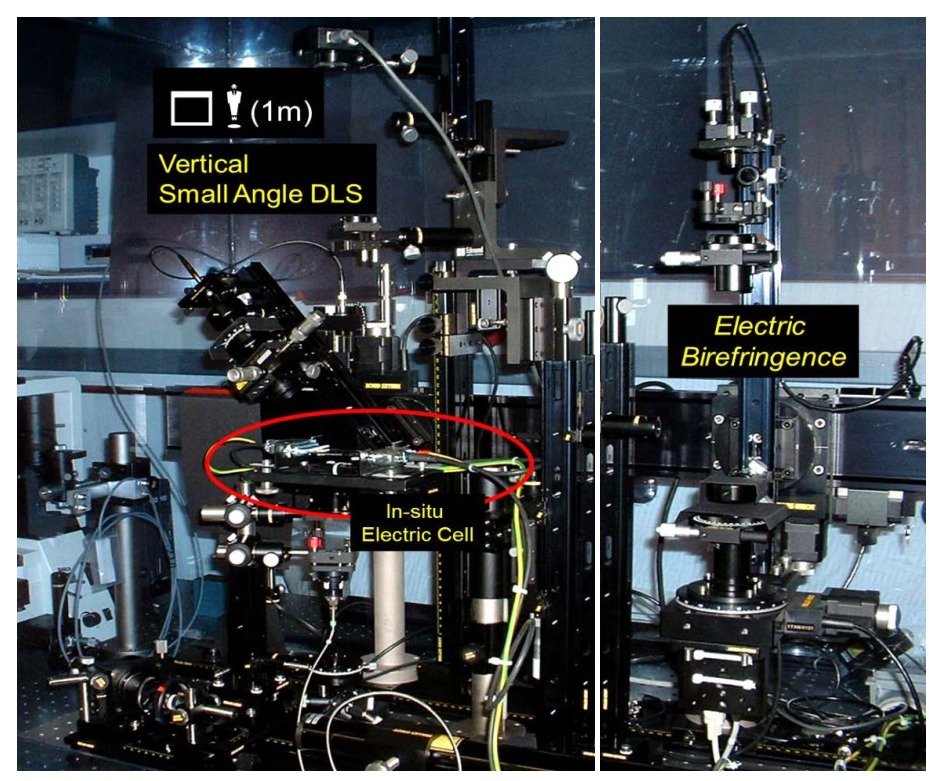

Figure 4. Experimental set up of vertical dynamic light scattering (left) and electric birefringence (right).

in Figure 4): two flat ITO coated glass surfaces, where the sample is held in between the two glass plates by capillary forces. For the higher fd-concentrations without the electric field, a commercially available cylindrical cuvette is used, instead of the electric-cell. On the detection side there is an achromatic lens with a focal length of $75 \mathrm{~mm}$ inserted, in order to select a scattering volume within the bulk of the sample, away from the two surfaces of the cell. Dynamic light scattering on approach of the critical point from the side of the $H$-phase reveals the development of a slow mode in the particle dynamics [8]. There are thus indications that, in additional to the texture dynamics in the dynamical state, also the particle dynamics slows down at the critical point. The dynamic light scattering set up is shortly described in the next subsection, which will also be used to probe the particle dynamics in Section 3 on the glass transition.

\subsection{Electric Birefringence and Orientation Orders}

The orientational nematic order is measured with a homemade electric birefringence setup, shown on the right in Figure 4, where the optical elements are aligned on the straight optical rail that can be rotated. Within the $H$ phase the rods are aligned along the electric field direction, so that the incident laser beam must be inclined to probe birefringence. What is measured is the transmitted intensity as a function of the angle $\alpha$ of the GlanThomson analyzer, which is related to the optical retardation $\Delta \phi$ as,

$$
I=\frac{1}{2} I_{\max }[1+\sin (2 \alpha+\Delta \phi)]
$$

With $I_{\max }$ the maximum transmitted intensity, and $\Delta \phi=2 \pi \Delta n_{\text {meas }} / \lambda$, where the measured birefringence is written as $\Delta n=\Delta n_{\text {meas }} / \sin ^{2} \delta$, for a given oblique incident angle of $\delta<20^{\circ}$ [2]. Thus the retardation is related to the birefringence as well as the angle of incidence. Hence, the determination of the analyzer angle where the minimum intensity is found allows the calculation of the birefringence, and though that the orientational order within the $H$-phase, as well as diffusion coefficients measured by dynamic light scattering, are independent of the field amplitude and frequency throughout the entire $H$-phase (with the exception of the area in pink in Figure 2, where pre-transitional nematic domains are present). Charge polarization becomes insignificant at frequencies higher than about $500 \mathrm{~Hz}-1 \mathrm{kHz}$. The $H$-phase is thus most probably stabilized by hydrodynamic interactions due to the field-induced electro-osmotic flow. This might be the reason why orientational order and particle dynamics in the $H$-phase is independent of the field amplitude and frequency. Here, the phase angle shift turned out to be increased with an increase of fd-concentration, which is expected due to the increase of aligning effect, towards the applied electric field direction. 


\section{Glass Transition in Concentration}

Let us now consider the slow dynamics of orientation texture in the absence of the electric field, for concentrations above the coexistence region. The same experimental methods are used for long waiting time observation. Figure 5 depicts the initial break up of shear-aligned birefringent domains, after filling the cuvette, and the equilibration up to long waiting times, 80 - $100 \mathrm{~h}$, confirmed by an image-time correlation [7] [8]. Here a commercially available cylindrical cuvette (diameter of $20 \mathrm{~mm}$ and thickness $1 \mathrm{~mm}$ ) is used and placed between the crossed polarizers. Here, a beam shutter is used to block the incident light in between CCD exposure in order to prevent heating for the long waiting time. In total 1008 frames are recorded, with a $600 \mathrm{~s}$ time-binning for a single image recording. Note that this is much longer time steps as compared to that of the field-induced dynamical states, where 200 frames with typical time steps were in the order of $0.3-1 \mathrm{~s}$. The dynamics of the orientation texture is again quantified by means of image-time correlation.

The texture dynamics in the equilibrated state is found to be independent of the fd-concentration up to the concentration where particle arrest is found. The slow but finite decay rates of image-time correlation functions above the glass transition is due to slow stress release of the shear aligned texture. Hence, particle arrest and freezing of the nematic texture occurs at the same concentration. Later waiting time of Figure 5 has shown clearly that the morphology has rapidly changed in relatively short time lapse (within $17 \mathrm{~h}$ ).

\subsection{Transient Behaviors of Repulsive Glasses}

A glass is defined as an out-of-equilibrium state of matter where the time needed to reach equilibrium is far beyond the experimental observation time. In particular, the diffusive dynamics of particles is arrested above the glass-transition concentration. A glass transition concentration in the fd-system at low ionic strength (corresponding to a Debye length of $27 \mathrm{~nm}$ ) is found for concentrations far above the isotropic-nematic coexistence region [11] [12]. The particle dynamics is found to become arrested at a concentration of about $11.7 \mathrm{mg} / \mathrm{ml}$, as evidenced by dynamic light scattering. Figure 6 shows an intensity trace of scattered light for a concentration just below the glass transition, revealing the very slow dynamics. The corresponding power spectrum is also shown in the lower panel in Figure 6. The amplitude of the power spectrum increases with increasing concentration, indicative for the slowing down of dynamics. Since the glass state is formed far above the isotropic-nematic coexistence region, the morphology of the system consists of nematic domains with different orientations. Structural arrest and freezing of the orientational texture are found to occur at the same concentration. In addition, interesting features of the "transient" behaviors of charged fd-rod glasses are exhibited as 1) initially longtime caging due to structural arrest (up to $100 \mathrm{~h}$ ), followed by 2) the propagation of a mechanical flow that is originated by the local microscopic stress release, and 3) finally entering to the long-time metastable glass state [12].

\subsection{Elastic Deformation and Chiral Features of Slow Dynamics}

With and without an external electric field, and for concentrations above the isotropic-nematic coexistence re-

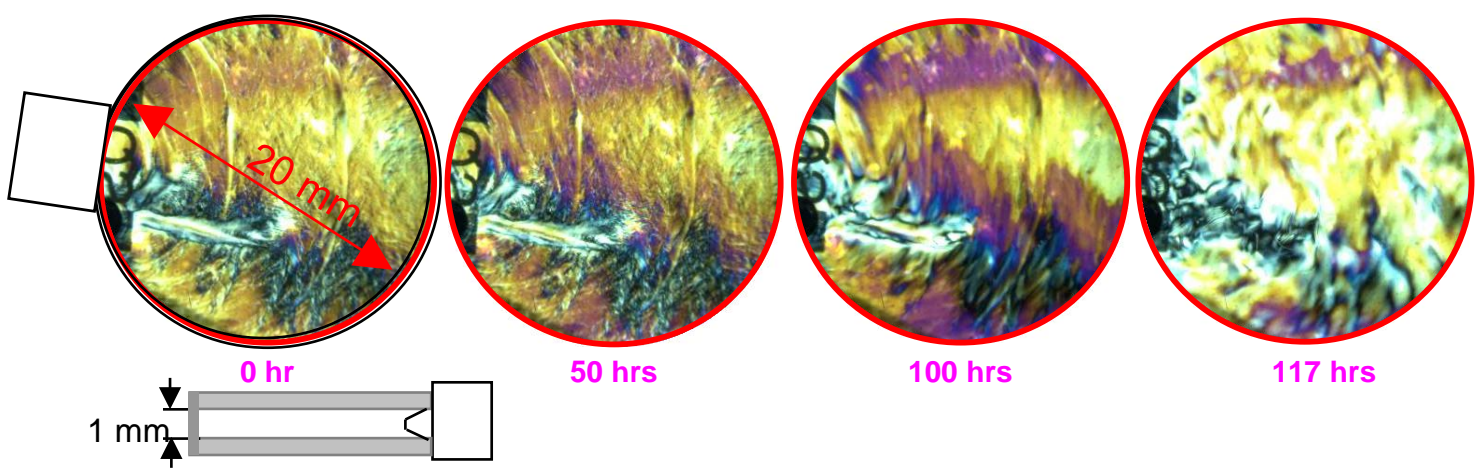

Figure 5. Depolarized optical morphologies of the glass, where the propagation of a flow is seen (the relatively dark areas are in flow). The first image is taken 117 hours after filling the cuvette. The white rectangle in the first image depicts the location of the entrance of the cuvette. The lower left sketch is a side view of the cylindrical cuvette. Its height is $1 \mathrm{~mm}$, while the cylindrical diameter is $20 \mathrm{~mm}$. 

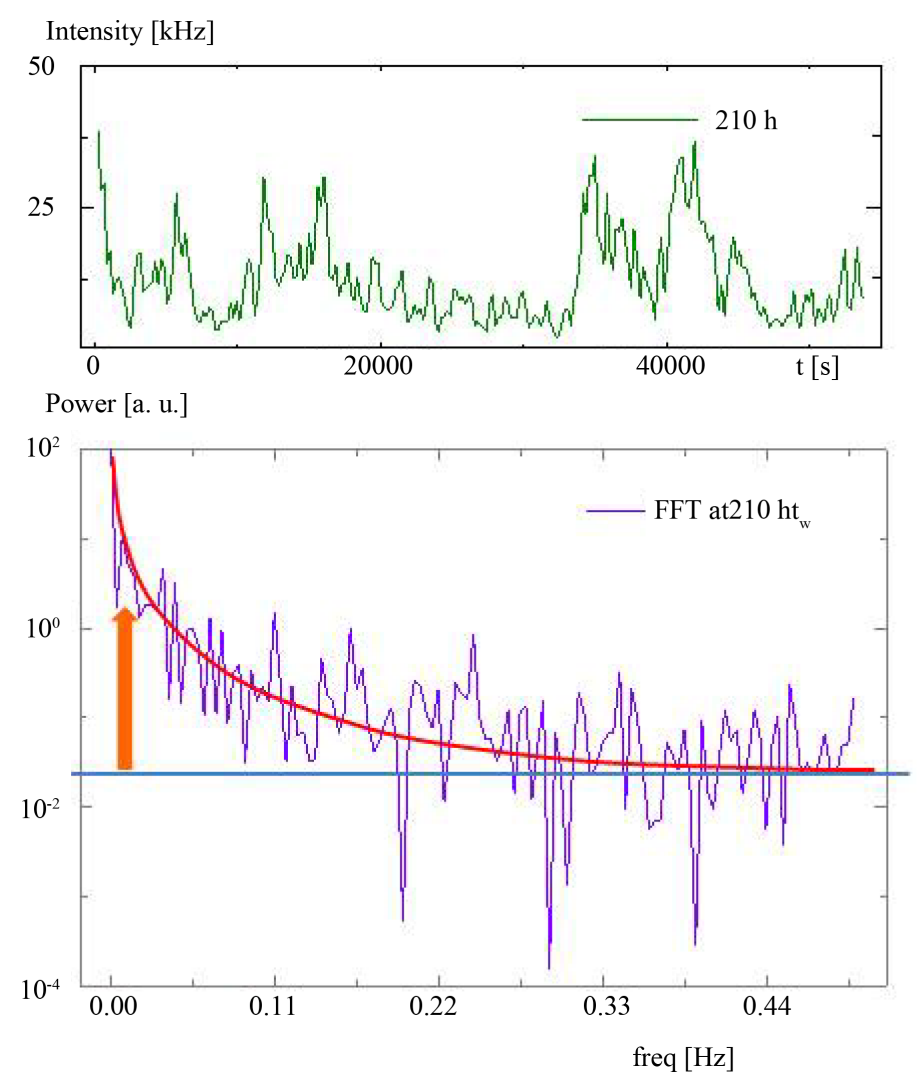

Figure 6. An intensity trace of scattered light for the charged rod glass concentration (upper panel). The lower panel shows the power spectrum of Fourier transform corresponding of the intensity distribution for longer waiting time of $210 \mathrm{~h}$ with duration time (of 58,000 s). The amplitude at zero frequency increases with fd-concentration, which signifies the approach of the glass transition.

gion, several texture morphologies are observed on increasing concentration (up to the glass concentration) in the depolarized images, shown in up and down in Figure 7, respectively. At low concentrations, just above the two-phase region, the nematic is non-chiral, as shown in the most left down image in Figure 7, in contrast with the nematic that is found at high salt concentrations (corresponding to Debye lengths of a few nano meters). For the low salt concentrations under consideration here (Debye length is $27 \mathrm{~nm}$ ), the long-ranged electrostatic repulsive interactions screen the chiral core-core fd-interactions. At somewhat higher concentrations, however, where the rod-rod distance becomes smaller, the chiral structure of the fd-core renders the nematic chiral (second image in Figure 7). The reason why in the presence of an electric field there is a transition a chiral nematic (see Figure 2) is due to the field-induced release of condensed ions, which renders the Debye length smaller, and therefore decreases the average distance between rods. The electric-field induced phases and states, illustrating dynamic frequency responses, are performed at the concentration of isotropic-nematic (I-N) coexistence: as a low frequency response, two sharp transitions of chiral-nematic phases and dynamical states are induced, while as one transition is found to a homeotropic phase that is stabilized at a high-frequency. The dynamics of particles and the orientation texture, below and above the glass transition, exhibits two dynamical modes, where the slow mode is attributed to the elasticity of helical domains, confirmed by dynamic light scattering correlation functions. On approach of the glass-transition concentration, the slow mode increases in amplitude, while as the amplitudes of fast and slow mode become equal at the glass transition [12]. In the case without the field, the increase of concentration renders the average distance between the rods smaller and thus induces chirality. At a higher concentration, the texture shows an X-pattern (third image in Figure 7). The dark blue regions are assumed as homeotropically aligned fd-rods. The boundaries of these "X-pattern" consist of 

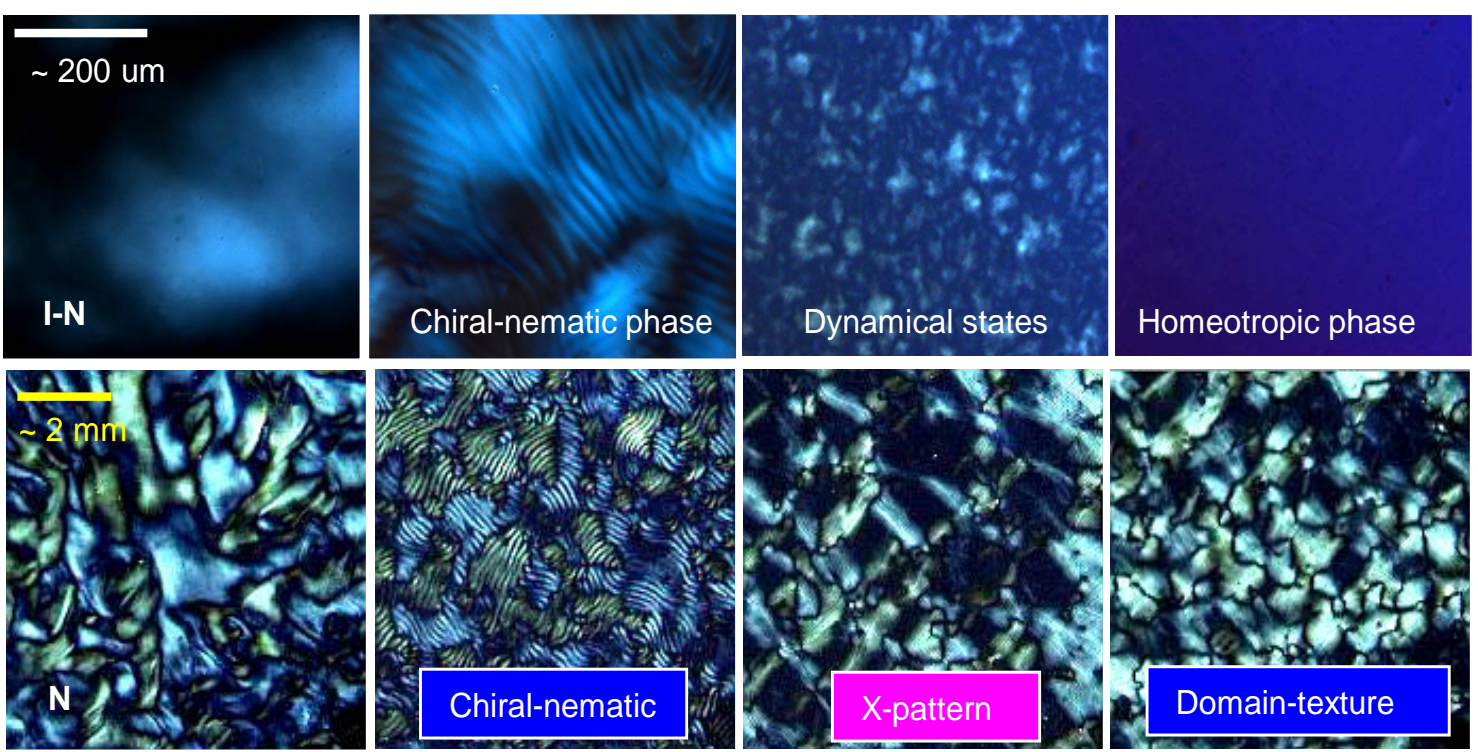

Figure 7. (Up) The comparison of depolarized morpholgy of electric-field induced phase/state of fd-concentration of 2.0 - $2.8 \mathrm{mg} / \mathrm{ml}$; and (Down) High-concentration orientation textures equilibrated for an initial-time (most left) and long waiting time, for fd-concentration of $3.8 \mathrm{mg} / \mathrm{ml}$ (chiral-nematic), $7.3 \mathrm{mg} / \mathrm{ml}$ (X-pattern), and $11.1 \mathrm{mg} / \mathrm{ml}$ (domain-texture) in the absense of external field.

helical domains. On even further increasing the concentration, the tightly packed domain texture is also consist of helical domains with twisted interfaces (most right image in Figure 7). It is as not yet clear why these phases form in the higher concentrations for long waiting time.

\section{Solitary Behaviors near the High-Frequency $H-N^{*}$ Phase Transition Line}

An interesting, soliton-like phase-separation kinetics is observed when the electric field frequency is suddenly reduced from the $H$-phase to just below the $N^{*}-H$-transition line. In the vicinity of the transition line, the chiral pitch of the $N^{*}$-phase is very large, and even pairs and isolated chiral streaks are seen. The most left image in Figure 8 shows the chiral nematic away from the transition line, while the second image illustrates the comparably very large pitch. Just inside the $H$-phase, within a region of about $100 \mathrm{~Hz}$ from the transition line, pretransitional domains appear. Time-resolved images after such a quench are shown in Figure 9. The chiral streaks move around within significant changes of their form, and thus behave like solitary waves. The formation of a quiescent state where temporal changes cease to occur takes several tens of minutes, contrary to the chiral nematic away from the transition line where the quiescent state is formed within fractions of seconds. As mentioned before, at frequencies around the $\mathrm{N}^{*}$-H-transition line, charge polarization interactions and hydrodynamic interactions due to field-induced electro-osmotic flow are approximately equally significant. Away from the transition line, within the $H$-phase hydrodynamic interactions are dominant, while within the $N^{*}$-phase interactions due to charge polarization are dominant. It might be that the interplay between these two types of interactions leads to the very slow kinetics and soliton-like behavior. Here, the system can be assumed as a weakly non-linear wave modulation by either inhomogeneities or ion concentration gradients, so that a propagation of wavefront within their own velocity is possible in a slow time variable. A collective polarization may become large enough to cause an elastic deformation to form arbitrarily large induced polarization for the pre-transitional large blurry nematic domains as spontaneous realigning effects, close to the $H-N^{*}$ phase transition. At relatively high frequency, the torque acting on the parallel direction to the applied field is found to have an opposite sign of the induced polarization of condensed ion (or charge) distribution [6], which leads to anomalous orientation to the perpendicular to the applied field. This sign change at high frequency is due to the "relatively" large contribution of out-of phase component of the torque. The rod-rod interactions resulting from field-induced polarization and possibly electro-osmotic flow are at the microscopic origin of the pre-transitional large blurry nematic domains as well as the observed elastic waves. 


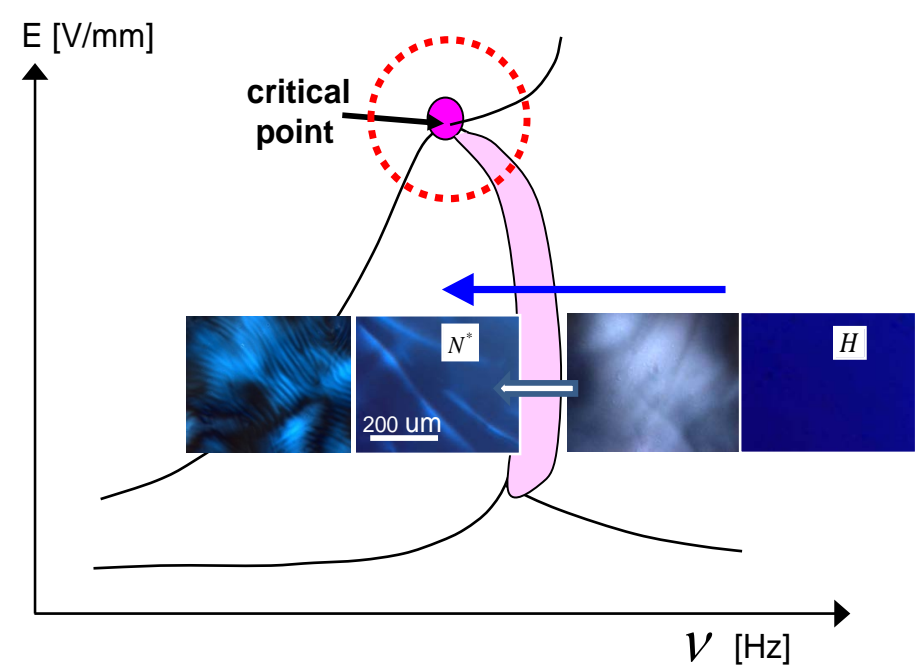

Figure 8. Depolarized images in various position in the phase/state diagram close to the $\mathrm{N}^{*}-\mathrm{H}^{-}$ transition line. The pink region indicates the region where pre-transitional, transient nematic domains are present (the third image).

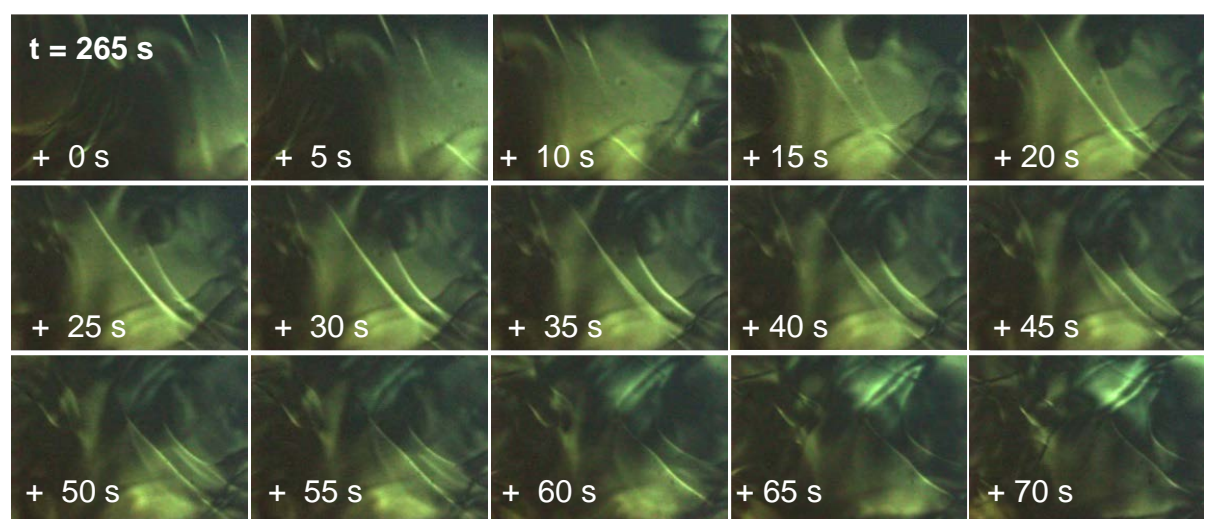

Figure 9. Time resolved depolarized images during the formation of the $N^{*}$-phase, more than $265 \mathrm{sec}$ after lowering the frequency, after a quench from the $H$-phase to the $N^{*}$-phase, close to the $H-N^{*}$ transition line. The chiral-stripes translate during the formation of the large-pitch $N^{*}$-phase without changing their shape.

\section{Acknowledgements}

Author K. K. thanks to Dr. H. Kriegs for the instrumentation development with an automation of homemade goniometer in small angle vertical dynamic light scattering setup.

\section{References}

[1] Kang, K. and Dhont, J.K.G. (2008) Double-Layer Polarization Induced Transitions in Suspension of Colloidal Rods. Europhysics Letters, 84, Article ID: 14005. http://dx.doi.org/10.1209/0295-5075/84/14005

[2] Kang, K. and Dhont, J.K.G. (2010) Electric-Field Induced Transitions in Suspensions of Charged Colloidal Rods. Soft Matter, 6, 273-286. http://dx.doi.org/10.1039/b910046f

[3] Zimmermann, K., Hagedorn, J., Heuck, C.C., Hinrichsen, M. and Ludwig, J. (1986) The Ionic Properties of the Filamentous Bacteriophages Pf1 and fd. Journal of Biological Chemistry, 261, 1653-1655.

[4] Manning, G.S. (2011) A Counterion Condensation Theory for the Relaxation and Frequency Dependence of the Polarization of Rod-Like Polyelectrolytes. The European Physical Journal E, 34, 1-7. http://dx.doi.org/10.1140/epje/i2011-11039-2

[5] Dhont, J.K.G. and Kang, K. (2011) Electric-Field-Induced Polarization of the Layer of Condensed Ions on Cylindrical 
Colloids. The European Physical Journal E, 34, 40. http://dx.doi.org/10.1140/epje/i2011-11040-9

[6] Dhont, J.K.G. and Kang, K. (2014) An Electric-Field Induced Dynamical State in Dispersions of Charged Colloidal Rods. Soft Matter, 10, 1987-2007. http://dx.doi.org/10.1039/c3sm52277f

[7] Kang, K. and Dhont, J.K.G. (2013) Glass Transition in Suspensions of Charged Rods: Structural Arrest and Texture Dynamics. Physical Review Letters, 110, Article ID: 015901. http://dx.doi.org/10.1103/PhysRevLett.110.015901

[8] Kang, K. and Dhont, J.K.G. (2014) Structural Arrest and Texture Dynamics in Suspensions of Charged Colloidal Rods. Soft Matter, 9, 4401-4411. http://dx.doi.org/10.1039/c3sm27754b

[9] Kang, K. (2011) Image Time-Correlation, Dynamic Light Scattering, and Birefringence for the Study of the Response of Anisometric Colloids to External Fields. Review of Scientific Instruments, 82, Article ID: 053903. http://dx.doi.org/10.1063/1.3589856

[10] Kang, K. (2010) Charged Fibrous Viruses (fd) in External Electric Fields: Dynamics and Orientational Order. New Journal of Physics, 12, Article ID: 063017. http://dx.doi.org/10.1088/1367-2630/12/6/063017

[11] Kang, K. and Dhont, J.K.G. (2009) Criticality in a Non-Equilibrium, Driven System: Charged Colloidal Rods (fd-Viruses) in Electric Fields. The European Physical Journal E, 30, 333-340. http://dx.doi.org/10.1140/epje/i2009-10525-4

[12] Kang, K. (2014) Glass Transition of Repulsive Charged Rods (fd-Viruses). Soft Matter, 10, 3311-3324. http://dx.doi.org/10.1039/c3sm53131g 
Scientific Research Publishing (SCIRP) is one of the largest Open Access journal publishers. It is currently publishing more than 200 open access, online, peer-reviewed journals covering a wide range of academic disciplines. SCIRP serves the worldwide academic communities and contributes to the progress and application of science with its publication.

Other selected journals from SCIRP are listed as below. Submit your manuscript to us via either submit@scirp.org or Online Submission Portal.
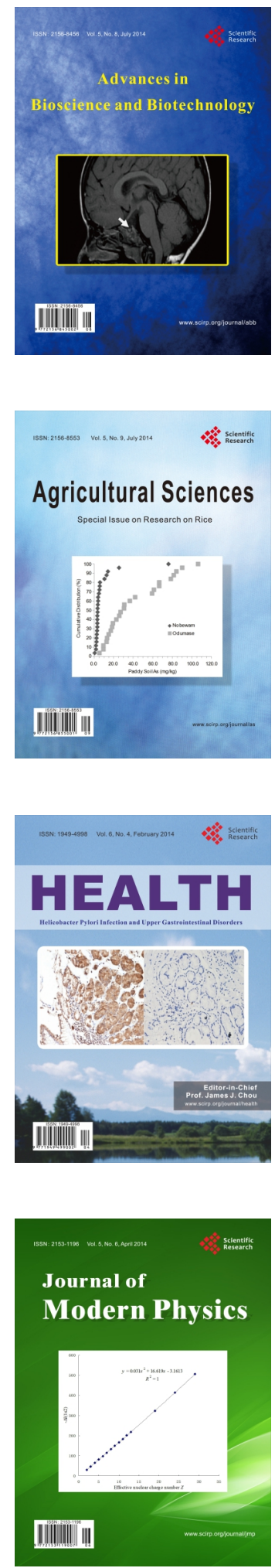
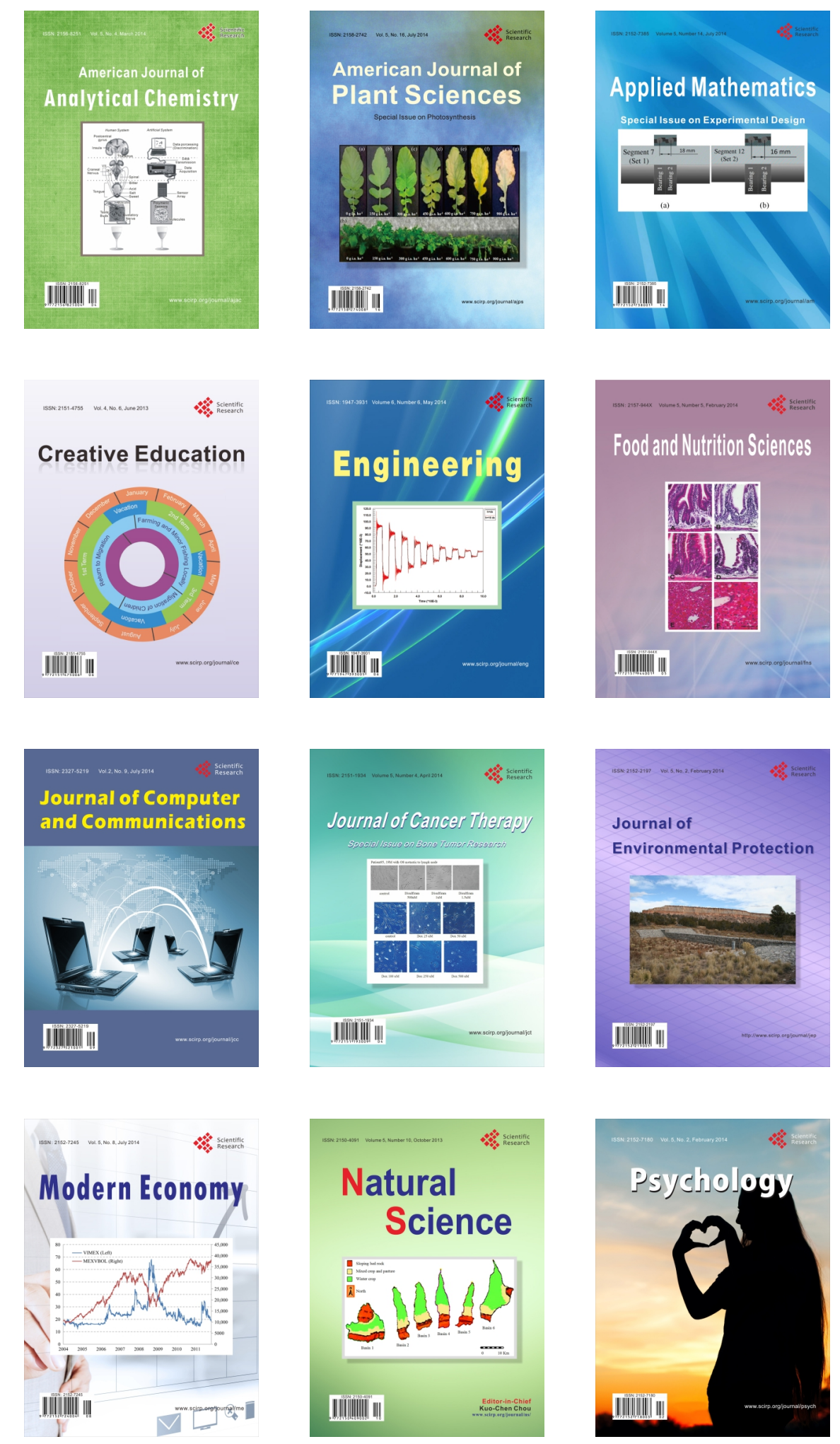